\title{
How to Assess Functional Significance of Myocardial Bridges in Athletes: A Personalized Medicine Approach
}

\author{
Leonarda Galiuto* \\ IOC dipl Sports Medicine, Department of Cardiovascular Sciences, A Gemelli University Hospital-IRCCS, Catholic University of \\ the Sacred Heart, Rome, Italy \\ *Corresponding author: Leonarda Galiuto, IOC dipl Sports Medicine, Department of Cardiovascular Sciences, A Gemelli \\ University Hospital-IRCCS, Catholic University of the Sacred Heart, Rome, Italy
}

ARTICLE INFO
Received: 幽 February 21, 2020
Published: March 02, 2020
Citation: Leonarda Galiuto. How to Assess
Functional Significance of Myocardial
Bridges in Athletes: A Personalized Medicine
Approach. Biomed J Sci \& Tech Res 26(2)-
2020. BJSTR. MS.ID.004313.

Keywords: Myocardial Bridges; Sports Medicine; Sports Cardiology; Athletes

\begin{abstract}
Myocardial bridging is a congenital coronary anomaly involving a segment of coronary artery coursing through myocardial wall, in a different depth and length, potentially affected by systolic compression. In athletes, such coronary anomaly is most often an incidental finding, due to increased use of high sensitivity noninvasive diagnostic tests. However, since myocardial bridge might potentially precipitate ischemia and even be responsible for sudden cardiac death, athletes need to be cleared for competition by an appropriate diagnostic test. Which is the ideal diagnostic test in such peculiar condition, in which the pathophysiological mechanism of ischemia is unknown and potentially different in different subjects, remains to be investigated. We focused on different diagnostic tests proposed and classified according to the most likely pathophysiological mechanism of ischemia in myocardial bridge. Accordingly, we analysed and discussed on ideal test in athletes with such condition.
\end{abstract}

Abbreviations: MB: Myocardial Bridge; IVUS: Intravascular Ultrasound; ICD: Intracoronary Doppler

\section{Introduction}

Myocardial bridge (MB), first mentioned by Reyman in 1737 [1], is the term that refers to muscle overlying the intramyocardial segment of an epicardial coronary artery. It is characterized by systolic compression of the tunneled segment and remains clinically silent in the majority of cases. The incidence of MB in pathologic series has been reported ranging between 15\% and $85 \%$ [2,3]; they are currently increasingly recognized in vivo due to the expansion of advanced non-invasive cardiovascular imaging techniques including computer tomography imaging and cardiovascular magnetic resonance [4], while in angiographic series such incidence ranges from $0.5 \%$ to $2.5 \%$ [5-7]. This large discordance suggests that only a minority of patients with MB are, in fact, at increased risk for clinical symptoms and cardiac events. Myocardial bridges are most commonly localized in the middle segment of the left anterior descending coronary artery. Diagonal and marginal branches may be involved in $18 \%$ and $40 \%$ of cases, respectively [8].

Clinical interest and systematic research have been triggered by an observed association of myocardial bridges with myocardial ischemia [8,9]. However, functional and anatomical characteristics of bridges able to precipitate ischemia as well as pathophysiological mechanisms of ischemia are still unclear. In fact, since myocardial blood flow is diastolic, while possible compression of muscle overlaying bridged coronary segment would occur in systole, in theory, myocardial bridges should not be responsible for myocardial ischemia. However, there are patients in whom myocardial bridges can be identified as the only source of ischemia [8] (Figure 1). It has been shown that diastolic myocardial compression may occur in $34-41 \%$ of bridged coronary vessel and that increased early diastolic flow velocity with changes in flow profile may be present 
in myocardial bridges [8]. Preliminary and anecdotal data suggest that coronary flow reserve may be reduced, being between 2 and 2.6 distally to myocardial bridges, as measured by diastolic fractional flow reserve [10], while microvascular flow reserve may be impaired in patients with myocardial bridges [11-13], with possible, but not constant, normalization after invasive or surgical treatment of the bridge [14-16]. Furthermore, vascular compression in myocardial bridges is not only systolic, but it persists in the early phase of diastole and it is associated with reduced coronary flow reserve [12].

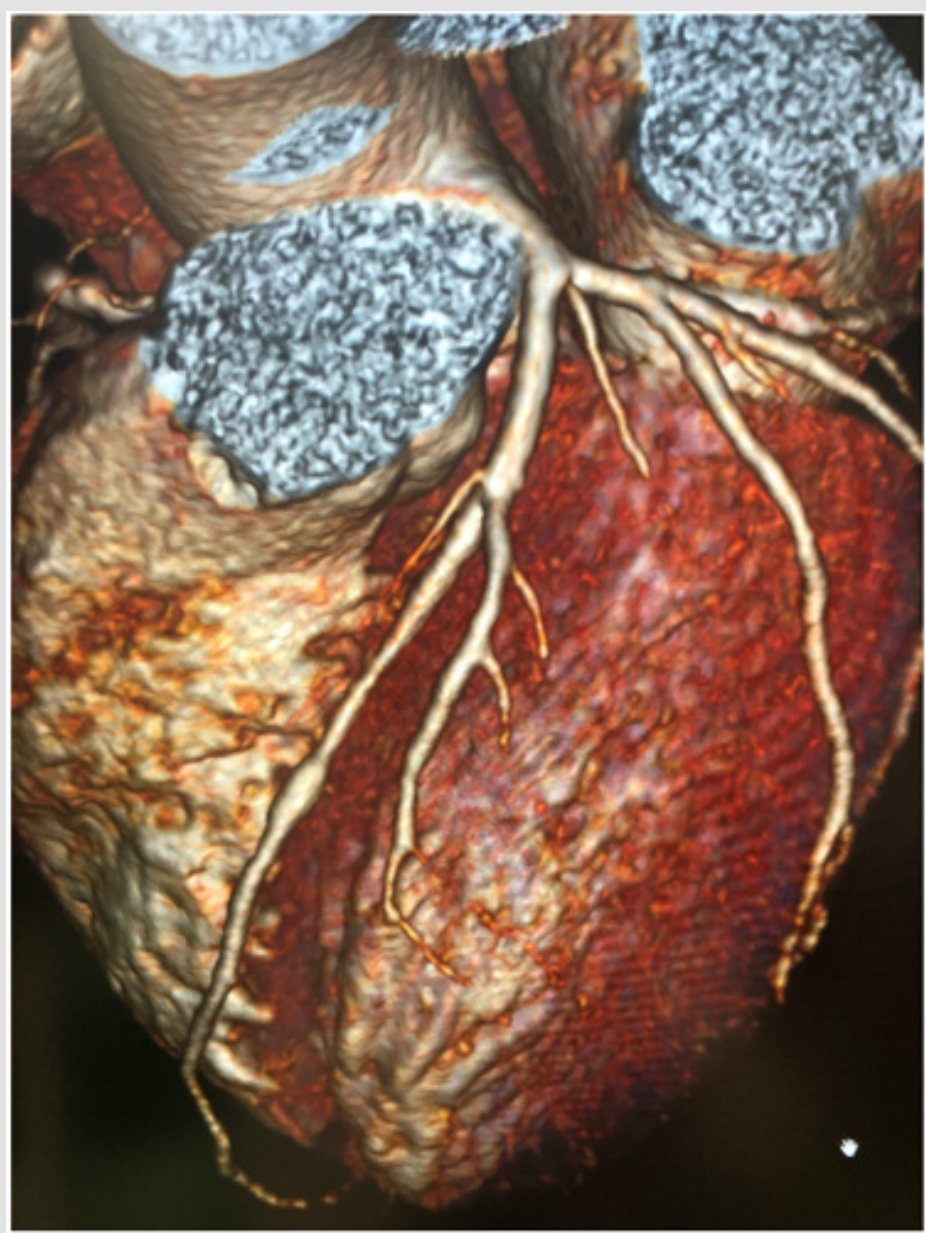

Figure 1: Myocardial bridge of middle LAD as diagnosed by coronary angio TAC (CT Force Siemens). Patients was referred for chest pain and developed a positive stress test.

Extravascular coronary compression by means of $\mathrm{MB}$ is modulated by myocardial inotropic force and causes intracoronary systolic pressure overshooting and negative systolic gradient across the MB, thus suggesting a possible role of increased inotropic stimulation as precipitating factor of ischemia in myocardial bridges [10,17]. Since the precise mechanism of myocardial ischemia in subjects with myocardial bridges is unknown and it may even be different according to the anatomy of the bridge and the characteristics of myocardial muscle, it derives that which is the best method to assess ischemia in such cases remains to be investigated and clarified. Anomalous coronary arteries have been documented in athletes [18] in whom the determination of the presence of such anomalies is crucial, since sudden death is associated with exercise $[19,20]$. Moreover, during exercise, diastole shortens due to increased heart rate with increased systolic/diastolic flow ratio and increased inotropic response produces not only systolic, but also diastolic compression, thus reducing flow. Lastly, de novo formation of myocardial bridges might occur in patients with hypertrophic cardiomyopathies [21] and in transplanted hearts [22], thus it is conceivable that they might develop also in athlete's heart.

The interest in diagnosing the presence and functional significance of bridges in athletes resides in the finding of MB as the only pathological findings in autopsies of subjects died for sudden cardiac arrest during sports [19,20]. Functional significance of myocardial bridges in athletes subjected to intense athletic training, reaching very high heart rates, undergoing adrenergic stimulation and possible myocardial hypertrophy, is even more complex to be assessed. An increase in sympathetic drive during stress or exercise likely facilitates ischemia, since tachycardia leads to an increase of systolic-diastolic time ratio at the expense of diastolic flow. Increased contractility during stress further aggravates systolic (and diastolic) compression. Endothelial dysfunction and coronary 
artery spasm may also contribute to constriction of the tunneled segment [23]. Furthermore, in athletes, functional implications of anomalous coronary arteries need to be clearly defined during the pre-participation screening in order to establish eligibility/ disqualification decisions in competitions [24].

The purpose of this point of view article is to focus and discuss on which is the ideal and most effective diagnostic method to reveal ischemia in athletes with myocardial bridges. Since modern medicine needs to be personalized, it is conceivable that, even in this field, appropriate diagnostic approach might reside on the identification of the mechanism of ischemia in each specific subject. Therefore, diagnostic potential in the detection of ischemia of different tests is discussed along the mechanism of ischemia that each of them is able to explore.

\section{Mechanisms of Ischemia and Diagnostic Tests}

Functional significance of myocardial bridges depends upon anatomical characteristics of myocardial bridges, namely the length and location of overlaying muscle, depth of the vessel and hypertrophy of the wall. Atherosclerosis of arterial wall proximal to the bridged coronary segment might also influence flow dynamic. Vasoconstriction and platelet aggregation might further reduce flow. Hemodynamic parameters, such as increased heart rate and reduced blood pressure, might be responsible for induction of ischemia. Therefore, since several pathophysiological mechanisms might be responsible for ischemia in myocardial bridges, we propose that diagnostic tests should be selected, according to the postulated mechanism of ischemia, based on the anatomical characteristics of the bridges and the functional status of the subject.

A. Coronary Diameter Reduction: Coronary angiography is an invasive diagnostic technique able to identify myocardial bridges based on the typical "milking effect" and a "step down - step up" phenomenon induced by systolic compression of the tunneled segment. Angiography is also able to diagnose the presence of atherosclerotic plaque formation, frequently involving the segment proximal to the bridge, with the tunneled segment typically spared. When bridges are diagnosed by invasive methods, morphological features can be visualized by intravascular ultrasound (IVUS), while functional characteristics can be measured by intracoronary Doppler ultrasound (ICD) and intracoronary pressure device [12]. IVUS analysis is able to show half-moon effect at rest or after provocation test, although its anatomical and physiological meaning are still not understood. Analysis of lumen area during the entire cardiac cycle can also be used to quantify the delay in relaxation after systolic compression. In ICD studies, flow pattern shows a sharp acceleration of flow in early diastole followed by immediate deceleration with a mid-diastolic plateau, as explained by an increased pressure gradient in early diastole, as a result of reduced distal coronary resistance. Occasionally, particularly in deep myocardial bridges, end-systolic flow reversal may precede rapid diastolic forward flow, as a result of local increase in pressure above aortic driving pressure [23].

Since vascular compression seems to be the major pathophysiological mechanism producing ischemia in subjects with myocardial bridge, dobutamine stress test might be proposed as the ideal test in such conditions, given the increased inotropic force produced by the drug. And in fact, a marked increase in flow velocity and an abnormal diastolic fractional flow reserve during dobutamine challenge has been demonstrated in symptomatic patients with focal wall motion abnormality upon exercise echocardiography [17]. Escaned, et al. [10] confirmed that inotropic stimulation is able to reveal the angiographic and functional severity of myocardial bridges, throughout the development of significant diastolic pressure gradients. Diastolic fractional flow reserve demonstrated to be superior to conventional fractional flow reserve, due to the exclusion for systolic pressure gradient. When dobutamine challenge is applied in the functional evaluation of myocardial bridges in athletes, chronotropic and inotropic response to dobutamine stimulation might be blunted due to betareceptor downregulation and/or desensitization typical of highly trained athletes [25]. Thus, dobutamine test, does not appear to be ideal stress test in athletes.

B. Increased Intracoronary Flow Velocities: Using intracoronary Doppler flow wire, resting average peak flow velocity and average diastolic peak velocity are higher in bridged coronary segment, as compared to the corresponding proximal and distal segment, with only minor changes in systolic flow. Mean diastolic/ systolic flow velocity ratio in different studies ranged between 2.4 to 2.9 8. In order to show abnormal flow profile and to diagnose induction of ischemia, atrial pacing can be performed during intracoronary Doppler flow velocity measurements. Atrial pacing produces increases in flow velocity, reduces diastolic plateau, increases systolic flow reversal. In myocardial bridges, during atrial pacing, increased heart rate is associated with abnormal or delayed relaxation in addition to shortened diastole, leading to alteration of flow possibly producing ischemic signs and symptoms.

C. Reduced Coronary Flow Reserve: Distally to a bridged myocardium, myocardial blood flow at rest is normal and vasodilator stress does not produce reduced myocardial perfusion. In patients studied by 15-oxygen water positron emission tomography, myocardial stress perfusion distal to myocardial bridging was comparable with remote control regions, with no differences in relation to systolic compression [25]. However, in other studies, perfusion imaging with Thallium-201 or Tc-99m SPECT with dipyridamole, demonstrated perfusion defect during stress [26,27]. The difference between the negative and positive studies resides in the percentage of myocardial bridge compression observed in study population. A threshold of 50 to $70 \%$ of systolic compression seems to be required to induce myocardial perfusion defect. 
D. Effect of Exercise: Exercise stress test is the most physiological method to assess ischemia, particularly in athletes, in whom the effect of training on cardiac function and flow needs to be assessed. In a series of athletes with myocardial bridges, exercise electrocardiogram appeared to be pathological demonstrating the presence of ischemia in such group of highly trained subjects [28]. If cardiac imaging is combined with exercise test, the diagnostic power of the test is significantly increased [29]. In fact, Italian guideline, state that in case of exercise ECG positive or nondiagnostic for ischemia, athletes should undergo exercise imaging test to verify the presence and extent of ischemia. Cardiac SPECT imaging studies can be effective for assessing ischemia in patients with angina and myocardial bridging. The evaluation of myocardial perfusion with dipyridamole SPECT imaging showed a good agreement with exercise stress SPECT imaging for the detection of ischemia in this group of patients [27].

Accordingly, in athletes, novel diagnostic echocardiographic quantitative methods, such as exercise strain, may further improve the diagnostic power. In fact, a series of 58 patients with hemodynamically significant $\mathrm{MB}$, as determined by invasive diastolic fractional flow reserve, have significantly lower change in septal longitudinal strain on exercise echocardiography, suggesting that exercise strain echocardiography may be a useful test for noninvasively assessing the hemodynamic significance of a MB [30].

\section{Conclusion}

Based on the anatomical and functional characteristics of the bridges and on the clinical presentation of patients, ischemic potential of MB con be evaluated by personalized test approach. However, in athletes, particularly if clearance for competition needs to be released, exercise stress test with an associated imaging modality seems to be the ideal and most appropriate test.

\section{Acknowledgement}

The author acknowledges Federica Palma for editorial support.

\section{Conflict of Interest}

The author has no conflict of interest to declare and no fund were utilized for this work.

\section{References}

1. Reyman HC (1737) Disertatio de vasis cordis propriis. Med Diss Univ Gottingen 1737: 1-32.

2. Geiringer E (1951) The mural coronary. Am Heart J 41: 359-368.

3. Ferreira AG, Trotter SE, Konig B, L V Décourt, K Fox, et al. (1991) Myocardial bridges: Morphological and functional aspects. Br Heart J 66(5): 364-367.

4. Ripley DP, Saha A, Teis A, Uddin A, Bijsterveld P, et al. (2014) The distribution and prognosis of anomalous coronary arteries identified by cardiovascular magnetic resonance: 15 years' experience from two tertiary centers. J Cardiovasc Magn Res 16: 34.

5. Noble J, Bourassa MG, Petitclerc R (1976) Myocardial bridging and milking effect of the left anterior descending coronary artery: Normal variant or obstruction? Am J Cardiol 37: 993-999.
6. Juillière Y, Berder V, Suty Selton C, Buffet P, Danchin N, et al. (1995) Isolated myocardial bridges with angiographic milking of the left anterior descending coronary artery: A long-term follow-up study. Am Heart J 129: 663-665.

7. Angelini P (2007) Coronary artery anomalies: An entity in search of an identity. Circulation 115: 1296-1305.

8. Bourassa MG, Butnaru A, Lespérance J (2003) Symptomatic myocardial bridges: Overview of ischemic mechanisms and current diagnostic and treatment strategies. J Am Coll Cardiol 41: 351-359.

9. Ural E, Bildirici U, Celikyurt U, Kilic T, Sahin T, et al. (2009) Long-term prognosis of non-interventionally followed patients with isolated myocardial bridge and severe systolic compression of the left anterior descending coronary artery. Clin Cardiol 32: 454-457.

10. Escaned J, Cortés J, Florex A, Goicolea J, Alfonso F, et al. (2003) Importance of Diastolic Fractional Flow Reserve and Dobutamine Challenge in Physiologic Assessment of Myocardial Bridging. J Am Coll Cardiol 42: 226-233.

11. Ge J, Jeremias A, Rupp A, Abels M, Baumgart D, et al. (1999) New signs characteristic of myocardial bridging demonstrated by intracoronary ultrasound and Doppler. Eur Heart J 20: 1707-1716.

12. Ge J, Erbel R, Rupprecht HJ, Koch L, Kearney P, et al. (1994) Comparison of intravascular ultrasound and angiography in the assessment of myocardial bridging. Circulation 89: 1725-1732.

13. Herrmann J, Higano ST, Lenon RJ, Rihal CS, Lerman A, et al. (2004) Myocardial bridging is associated with alteration in coronary vasoreactivity. Eur Heart J 25: 2134-2142.

14. Klues HG, Schwarz ER, Vom Dahl J, Reffelmann T, Reul H, et al. (1997) Disturbed intracoronary hemodynamics in myocardial bridging: early normalization by intracoronary stent placement. Circulation 96- 29052913.

15. Pratt JW, Michler RE, Pala J (1999) Minimally invasive coronary artery bypass grafting for myocardial muscle bridging. Heart Surg Forum 2(3): 250-253.

16. Kunamneni PB, Rajdev S, Krishnan P, Moreno PR, Kim MC, et al. (2008) Outcome of intracoronary stenting after failed maximal medical therapy in patients with symptomatic myocardial bridge. Catheter Cardiovasc Interv 71: 185-190.

17. Lin S, Tremmel JA, Yamada R, Rogers IS, Yong CM, et al. (2013) A Novel Stress Echocardiography Pattern for Myocardial Bridge with Invasive Structural and Hemodynamic Correlation. J Am Heart Assoc 2: 1-11.

18. Pelliccia A, Spataro A, Maron BJ (1993) Prospective echocardiographic screening for coronary artery anomalies in 1360 elite competitive athletes. Am J Cardiol 72: 978-979.

19. Morales AR, Romanelli R, Boucek RJ (1980) The mural left anterior descending coronary artery, strenuous exercise and sudden death. Circulation 62: 230-237.

20. Corrado D, Basso C, Rizzoli G, Schiavon M, Thiene G, et al. (2003) Does the sports activity enhance the risk of sudden death in adolescent and young adults? J Am Coll Cardiol 42: 1959-1963.

21. Vongpatanasin W, Willard JE, Hillis LD, Lange RA, Landau C, et al. (1997) Acquired myocardial bridging. Am Heart j 133: 463-465.

22. Jain SP, White CJ, Ventura HO (1993) De novo appearance of myocardial bridge in heart transplant: assessment by intravascular ultrasonography, Doppler and angioscopy. Am Heart J 126: 453-456.

23. Mohlenkamp S, Hort W, Ge J, Erbel R (2002) Update on myocardila bridging. Circulation 106: 2616-2622.

24. Pelliccia A, Fagard R, Bjørnstad HH, Anastassakis A, Arbustini E, et al. (2005) Study Group of Sports Cardiology of the Working Group of Cardiac Rehabilitation and Exercise Physiology; Working Group of Myocardial and Pericardial Diseases of the European Society of Cardiology Recommendations for competitive sports participation in athletes with 
cardiovascular disease. A consensus document from the Study Group of Sports Cardiology of the Working Group of Cardiac Rehabilitation and Exercise Physiology, and the Working Group of Myocardial and Pericardial diseases of the European Society of Cardiology. Eur Heart 26: 1422-1445.

25. Welsh RC, Warburton DER, Humen DP, Dylan A Taylor, Jonathon Mc Gavock, et al. (2005) Prolonged strenuous exercise alters the cardiovascular response to dobutamine stimulation in male athletes. J Physiol 569(Pt 1): 325-330.

26. Gawor R, Kuśmierek J, Płachcińska A, Bieńkiewicz M, Drożdż J, et al. (2011) Myocardial perfusion GSPECT imaging in patients with myocardial bridging. J Nucl Cardiol 18: 1059-1065.

27. Lee YS, Moon DH, Shin JW, Park SW, Park SJ, et al. (1999) Dipyridamole Tl-201 SPECT imaging in patients with myocardial bridging. Clin Nucl Med 24(10): 759-764.

ISSN: 2574-1241

DOI: $10.26717 /$ BJSTR.2020.26.004313

Leonarda Galiuto. Biomed J Sci \& Tech Res

(C) This work is licensed under Creative

Submission Link: https://biomedres.us/submit-manuscript.php
28. Sperandii F, Guerra E, Tranchita E, Minganti C, Lanzillo C, at al. (2017) Clinical significance of ST depression at exercise stress testing in competitive athletes: Usefulness of coronary CT during screening. The journal of sports Medicine and Physical Fitness 58(12):1876-1882.

29. Iliceto S, Galiuto L, Marangelli V (1994) Review Clinical use of stress echocardiography: factors affecting diagnostic accuracy. Eur Heart J 15(5): 672-680

30. Kobayashi Y, Tremmel JA, KobayashiY, Amsallem M, Tanaka S, et al. (2015) Exercise Strain Echocardiography in Patients with Hemodynamically Significant Myocardial Bridge Assessed by Physiological Study. J Am Heart Assoc 4: e002496.

$\begin{array}{ll}\text { BIOMEDICAL } & \text { Assets of Publishing with us } \\ \text { RESEARCHES } & \text { - Global archiving of articles } \\ & \text { - Immediate, unrestricted online access } \\ & \text { - Rigorous Peer Review Process } \\ & \text { - Anttps://biomedres.us/ }\end{array}$

